\title{
Working Memory and Lexical Ambiguity Resolution as Revealed by ERPs: A Difficult Case for Activation Theories
}

\author{
Thomas C. Gunter, Susanne Wagner, and Angela D. Friederici
}

\begin{abstract}
This series of three event-related potential experiments explored the issue of whether the underlying mechanism of working memory (WM) supporting language processing is inhibitory or activational in nature. These different cognitive mechanisms have been proposed to explain the more efficient processing of subjects with a high WM span compared to those with a low WM span. Participants with high and low WM span were presented with sentences containing a homonym followed three words later by a nominal disambiguation cue and a final disambiguation using a verb. At the position of the disambiguation cue, inhibitory or activational WM mechanisms predict contrasting results. When activation is the underlying mechanism for efficient processing, the prediction is that high memory span persons activate both meanings of the homonym equally in WM, whereas low memory span persons only have one meaning present. When inhibition is the underlying mechanism, the
\end{abstract}

\section{INTRODUCTION}

Recent studies in psycholinguistics indicate that there is a strong interaction between working memory (WM) and language processing (Vos, Gunter, Kolk, \& Mulder, 2001; Vos, Gunter, Schriefers, \& Friederici, 2001; Friederici, Steinhauer, Mecklinger, \& Meyer, 1998; Gunter, Jackson, \& Mulder, 1995; King \& Just, 1991; King \& Kutas, 1995). There is, however, considerable disagreement about the mechanisms underlying WM employed during language processing (see also Engle, 1996); while some behavioral experiments demonstrate evidence supporting an activation mechanism (i.e., activation of relevant information; cf. Miyake, Just, \& Carpenter, 1994; Just \& Carpenter, 1992), others propose an inhibition mechanism (i.e., suppression of irrelevant information; cf. Friederici et al., 1998; Gernsbacher \& Faust, 1991). The present study proposes to disentangle this issue by measuring brain activity using event-related

Max Planck Institute of Cognitive Neuroscience predictions are the reverse. The ERP data, in particular, the variations of the meaning related N400 component, showed clear evidence for inhibition as the underlying cognitive mechanism in high-span readers. For low-span participants the cueing towards the dominant or the subordinate meaning elicited an equivalently large N400 component suggesting that both meanings are active in WM. In highspan subjects, the dominant disambiguation cue elicited a smaller N400 than the subordinate one, indicating that for these subjects particularly the dominant meaning is active. The experiments showed that inhibitory processes are probably underlying WM used during language comprehension in high-span subjects. Moreover, they demonstrate that these subjects can use their inhibition in a more flexible manner than low-span subjects. The effects that these processing differences have on the efficiency of language parsing are discussed. potentials (ERPs) in persons with high or low WM capacity during the processing of sentences containing an ambiguous word.

A major issue in the psychology of language relates to the nature of the interaction of (working) memory and the processing of complex language materials. It is clear that in sentences containing an embedded clause, WM plays an important role, particularly during the processing of the postembedded part (in Dutch or German at the sentence final verb; cf. Gunter et al., 1995). In general, it is found that such complex material is more efficiently processed by subjects with a larger WM capacity. Research on the interaction of WM capacity with syntactic complexity/ambiguity (i.e., subject relative vs. object relative clauses) has revealed, for instance, that individuals with a high WM span are more efficient in parsing than those with a low WM span (Fiebach, Schlesewsky, \& Friederici, 2001; Vos et al., 2001; Friederici et al., 1998; Mecklinger, Schriefers, Steinhauer, \& Friederici, 1995).

In cognitive science, there are two major hypotheses currently entertained concerning the underlying 
mechanisms of WM use in language processing. One hypothesis claims that the involvement of WM in reading language input efficiently relates to "activation" processes. This view has been formulated for the processing of syntactically ambiguous material (Just \& Carpenter, 1992; King \& Just, 1991) as well as for the processing of lexical ambiguity (Miyake et al., 1994). A person with a high WM capacity is assumed to activate both readings of an ambiguity, whereas a person with low WM capacity is taken to activate only one reading. The alternative hypothesis holds that an "inhibition" mechanism in WM plays a crucial role in efficient language processing. This cognitive mechanism has been argued to affect the efficient processing of both syntactic ambiguities (Friederici et al., 1998) and lexically ambiguous material (Gernsbacher \& Faust, 1991). Under this view, a person with a high WM span is able to suppress (i.e., inhibit) irrelevant information more effectively than a person with a low WM span, thus relating the differences observed to a different use of the WM capacity. Note that the same predictions can be made on the assumption that the second reading is not inhibited completely, but that there is a ranking in the activation of both readings with the relevant information receiving higher activation than the irrelevant information. Such a proposal has been advanced for the processing of syntactically ambiguous sentences (Hickok, 1993). As the ranked and the inhibition views make the same predictions with respect to fast on-line processes, we will treat them alike.

At first sight, the activation-inhibition distinction may seem trivial. In specific cases, however, the choice of the underlying mechanism leads to completely different predictions. One such case is the processing of homonyms (for a review, see, for instance, Simpson, 1994). A homonym is a word which has two meanings but only one spelling and pronunciation (e.g., boxer: sportsman or dog). Typically, a homonym has a more expected dominant meaning and a lesser expected subordinate meaning. Cases where both meanings are equally expected are rare. Studies using the crossmodal priming paradigm show that, even if the preceding context favors only one interpretation, both interpretations are activated immediately after the occurrence of the homonym (Swinney, 1979). Typically, this multiple-access phase occurs within approximately 200 msec followed by a meaning selection phase in which the contextually appropriate interpretation is selected and the inappropriate interpretation decays or is actively suppressed. ${ }^{1}$ Although some behavioral studies seem to indicate that the multiple-access phase is relatively short lived, others (i.e., Miyake et al., 1994) show that this is not always the case, but that its duration seems to depend on whether or not meaning selection is needed. When a homonym is presented in a neutral context, such as in the following example from Miyake et al. (1994), readers cannot make a correct meaning selection until the eighth word after the presentation of the homonym ${ }^{2}$ :

\section{Since Ken really liked the boxer, be took a bus to the nearest pet store to buy the animal. \\ sports arena to see the match.}

This paradigm provides a forum for the resolution of the question concerning the notion of the mechanisms underlying WM when used in conjunction with lowand high-WM span readers: If activation plays the major role in WM and, as suggested by the Just and Carpenter group, high-span readers have better activational resources, one must expect high-span readers to have both meanings still present in WM at the disambiguation point. Low-span readers, on the other hand, will be predicted to only have the dominant meaning present at this point. If inhibition is the underlying mechanism of WM, one would expect low-span readers to have both meanings present to a much larger extent than high-span readers who will suppress the subordinate meaning because it is the less probable one. It is clear that these competing mechanisms predict totally different results.

Neuroscience, so far, has contributed only little to this debate. Although there are a number of patients and imaging studies on the interplay of WM and sentence processing (Cooke et al., 2001; Fiebach et al., 2001; Caplan \& Waters, 1999; Stowe et al., 1998; Just, Carpenter, Keller, Eddy, \& Thulborn, 1996), none of these has focused on the issue of high- versus lowspan subjects. All of these studies suggest that Broca's area is crucially involved in the processing of syntactically complex and memory-demanding sentences. While some researchers argue that there are different types of WM in language comprehension (i.e., a syntactic and a general one; see Caplan \& Waters, 1999), others view WM as a unified cognitive component (Just \& Carpenter, 1992).

The issue of sentence processing in high- and lowspan subjects has recently been addressed in a number of experiments (Vos \& Friederici, 2003; Vos et al., 2001; Friederici et al., 1998), which employ time-locked electrical brain activity, the so-called event-related potentials (ERPs). ERPs are thought of as providing an on-line measure of cognitive processing with a high temporal resolution. The cited studies, which focused on the interaction between syntactic complexity and WM span, indicate different parsing strategies in highand low-span readers. High-span readers appear to activate only one reading of a syntactically ambiguous sentence, independent of both the preceding semantic context (Mecklinger et al., 1995) and of intersentential syntactic context (Vos \& Friederici, 2003). Low-span readers, in contrast, appear to activate on-line both readings to some extent and only use intersentential context in an off-line manner (Vos \& Friederici, 2003). These syntax-oriented studies have used the presence 
of syntax-related ERP components, namely, the P600, to indicate whether only one sentence structure has been activated or not. The present study uses another ERP component, the N400, as a marker of how lexical ambiguity is processed during sentence comprehension in high- and low-span subjects.

The N400 component, which has a negative amplitude and a latency of approximately $400 \mathrm{msec}$, has been found to be sensitive to lexical semantic processes (Kutas \& Hillyard, 1980; for a review, see Kutas \& Federmeier, 2000; Van Petten, 1995). For the present experiments, it is important to note that when a word does not fit the semantic context of a sentence, the N400 is larger than when it fits the context. Phenomena like this have led researchers to suggest that the N400 must be associated with semantic integration processes (Brown \& Hagoort, 1993).

It appears that such integrative processing must be done within the WM system because several sources of linguistic information must be simultaneously present and/or active for integration to take place. Thus, when an item is easy to integrate, it must have been activated (i.e., be present) in WM. Consider the abovementioned boxer example. If both meanings of the homonym are (still) present in WM by the time that the disambiguating target words are encountered, both "pet store" and "sports arena" should be equally easy to integrate into the sentence. In such a case, both target items should elicit similarly large N400 components. Suppose, however, that the subordinately related target word elicits a larger N400 than the dominantly related target. Such a finding would indicate that the subordinately related target word is more difficult to integrate than the dominant one, which in turn would suggest that the subordinate meaning was inhibited/less present in WM. We assume that this difference in integration difficulty is due to differences in activation level in WM of the target words. The target, which is more difficult to integrate, has a smaller activation level in WM than the easy one (note that semantic expectancy cannot play a role here since the context of the boxer example is neutral). To state it differently, when a large N400 is found for a given target word, this means that the related meaning of this word is less present in WM than when a small N400 is found. A small N400 therefore indicates that the relevant meaning of the target word is active in WM.

Only a few ERP studies have looked specifically at the processing of homonyms. Van Petten and Kutas (1987) presented subjects with sentences which ended with a homonym. Following each sentence, a target was presented which was either related to the contextually biased meaning, related to the unbiased meaning, or unrelated to either meaning. When the stimulus onset asynchrony (SOA) was $700 \mathrm{msec}$, only the contextual appropriate meaning showed a reduced N400 component. When the SOA was $200 \mathrm{msec}$, both the context- appropriate and -inappropriate targets showed an N400 reduction although there was a later onset for the inappropriate ones. From these data, the authors concluded that both meanings of an ambiguous word are not activated at the same time.

A more recent study of Hagoort and Brown (1994) explored the processing of homonyms in the middle of a sentence. Subjects were presented with Dutch sentences which contained a neutral context, an ambiguous word, followed two words later by a disambiguating context. Compared to a neutral control word, the processing of the ambiguous word elicited a frontal slow shift which started after approximately $300 \mathrm{msec}$. One interpretation of this shift is that it could reflect processing costs related to the multiple access or alternatively to the costs associated with the computation of different higher message representations (i.e., multiple integrations). At the point of disambiguation, the word associated with the dominant meaning did not show a significant difference compared to a control word. The word associated with the subordinate meaning, however, showed a larger N400 compared to control words. As suggested by Hagoort and Brown, these results are compatible with the integration model of Rayner and Frazier (1989) and the reordered access model of Dopkins, Morris, and Rayner (1992). These models assume that when an ambiguous word is encountered in a neutral context, the parser integrates the dominant meaning initially because it receives more activation during a multiple-access phase. If subsequent information is pointing towards the subordinate meaning, reanalysis processes are invoked.

Swaab, Brown, and Hagoort (1997) investigated the auditory processing of ambiguous words in Broca's aphasics and reported evidence for a separate selectional process. Although the aphasic subjects had both meanings activated, they were not able to select the context-appropriate one within $100 \mathrm{msec}$. When given more time (i.e., $1250 \mathrm{msec}$ ), they did select the context-appropriate meaning. For healthy subjects, in contrast, a selection was reported for both ISIs. For the purpose of the present experiment, it is important to note that ERPs are sensitive towards homonym processing and that the N400 component can serve as a tool in this respect.

In the present series of experiments, the inhibition/ activation issue is approached in the following way. Subjects are visually presented with sentences in which the first part consists of an ambiguous noun phrase, an auxiliary and a case-marked preposition (Der Ball wurde vom, The ball was by the). Next, a cue towards either the dominant or subordinate meaning was presented by using a noun (i.e., Spieler [player] or Tänzer [dancer]). Occurrence of this disambiguation cue at this point serves essentially as a probe of WM. If both meanings of the sentence initial homonym are equally present at this point of time, the sentential integration of either 
noun should be equally difficult/easy, resulting in no N400 differences between the two disambiguation cues. ${ }^{3}$ However, if only one meaning is present in WM (i.e., the dominant meaning), the noun related to that meaning will be easier to integrate into the sentence than the noun related to the subordinate meaning. This should lead to a larger N400 component for the subordinate noun. Thus, the effect observed at the disambiguation cue will provide evidence which should help resolve the inhibition/activation issue. If high-span subjects show an N400 difference between dominant and subordinate disambiguations at the disambiguating cue, and low-span subjects do not, inhibition of the subordinate meaning after exhaustive lexical access is the more likely source underlying the reported processing superiority of high-span subjects. If low-span subjects show an N400 difference between the disambiguations but high-span subjects do not, then it is likely that the high-span subjects are able to activate both meanings, but low-span subjects cannot, and hence, WM is driven via activation.

One other important aspect of WM is the flexibility with which this resource can be used. In order to further explore this flexibility, the sentences (Der Ball wurde vom Spieler/Tänzer; The ball was by the player/dancer) were then continued with a verb which led to the final disambiguation toward either the dominant (i.e., geworfen; thrown) or subordinate meaning (eröffnet; opened). The flexibility of WM was investigated using so-called switch trials where the disambiguation cue and the final disambiguation did not match. A switch between the dominant and the subordinate meaning would be: Der Ball wurde vom Spieler eröffnet (The ball was by the player opened). When WM is used in a flexible and efficient manner, switching should be independent of direction. That is, switching from dominant to subordinate meanings should be as easy as switching from subordinate to dominant meanings. If inhibition is the underlying mechanism in high-span readers as compared to low-span readers, one would expect high-span readers but not low-span readers to use the disambiguation cue to greatly inhibit the "other" meaning. High-span subjects should therefore show a much larger N400 effect than low-span individuals at the final disambiguating verb in those cases in which the disambiguation cue and final disambiguations do not match. If, on the other hand, activation is the underlying mechanism of WM, high-span persons will have both meanings still active in memory even after the disambiguation cue and switch effortless between the two meanings. Low-span persons will use the disambiguation cue to relieve memory load and will thus have a more effortful switch because only one meaning is highly active. In terms of the ERP effects, this means that lowspan persons will show large N400 effects in "switch" trials at the final verb disambiguation, whereas high-span persons will not.

\section{EXPERIMENT 1: SWITCHING COSTS}

\section{Results}

\section{Percentage Correct}

Overall, participants performed well on the postsentential task since they reached a mean of $96 \%$ correct. The analysis across all subjects showed a main effect of switch indicating that question answering after the occurrence of a switch condition was less correct [switch: $95 \%$ vs. noswitch: 97\%; $F(1,31)=8.4, p<.007]$. A main effect of dominance indicated that questions were answered more correctly in the dominant condition [dominant: 97\% vs. subordinate: $95 \% ; F(1,31)=10.0, p<.0034$ ]. The analysis carried out on the subgroup of high- and low-span subjects showed main effects of switch and of dominance [switch: $F(1,22)=5.04, p<.06$; dominance: $F(1,22)=8.64, p<.008]$ but did not reveal a group main effect $[F(1,22)=0.01, n s]$ nor any interactions with group. Thus, high- and low-span subjects had a similar performance on the postsentential task.

\section{ERPs on the Disambiguation Cue}

As can be seen in the upper panel of Figure 1 where the ERP data across all 32 subjects are presented, the cue associated with the dominant meaning elicited a smaller N400 than the cue which made a "disambiguation" towards the subordinate meaning of the homonym. In other words, it seems that the dominant meaning of the homonym is present to a larger extent in WM than the subordinate meaning at the position of the disambiguation cue. These effects were confirmed by the latency window analysis, which showed clear noun main effects for all electrodes [i.e., Fz, C3, Cz, C4, and Pz; $F(1,31)=7.28-13.3]$.

The middle and lower panels of Figure 1 display the results for the low- and high-span subjects. Although it appears that only the high-span subjects have a small N400 effect, whereas the low-span participants do not

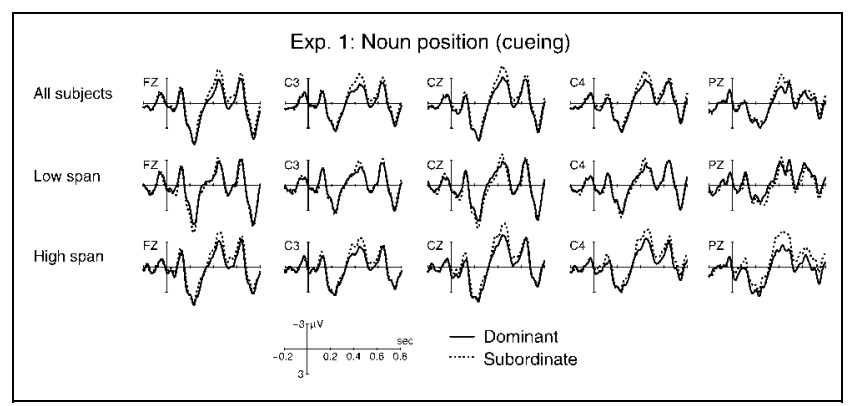

Figure 1. ERPs for the noun position of Experiment 1 where the disambiguation cue was given. The solid line shows the ERPs elicited by the dominant cueing, whereas the dashed line shows the subordinate cueing. The upper panel presents the data across all participants, the middle panel of the 12 low-span participants, and the lower panel of the 12 high-span participants. 
show an N400 difference, these group differences were not statistically significant. No noun with spangroup interaction was found in the main analysis of the data. As one will see in Experiment 2, however, this insignificance is due to the number of participants in both groups not providing enough statistical power to validate the relatively small N400 effects (approximately $0.5 \mu \mathrm{V}$ ).

\section{ERPS on the Verb}

As can be seen in Figure 2A and B, switching from one meaning to another meaning had a clear impact on the ERPs in that the N400 for the "switch" condition was larger compared to the "nonswitch" condition. This finding was confirmed by the statistical analysis which showed clear and significant main effects of "switch" [i.e., Fz, C3, Cz, C4, and Pz; F(1,31) = 16.13-31.77]. The analysis also showed a significant "switch" effect with dominance interactions [i.e., Fz, C3, Cz, C4, and Pz; $F(1,31)=4.46-16.11$ ]. It is clear from Figure 2 that the switch from the dominant towards the subordinate meaning (DD vs. DS) led to a much larger N400 difference than the switch from subordinate to dominant (SS vs. SD). This observation was confirmed by separate analyses carried out on DD versus DS and SS versus $\mathrm{SD}$. In the $\mathrm{DD}$ versus $\mathrm{DS}$ analysis, robust and significant "switch" main effects were found for all tested electrodes [Fz, C3, Cz, C4, and Pz; $F(1,31)=$ 21.24-38.76]. The SS versus SD analysis showed significant "switch" effects only for the C4 and Pz electrodes $[F(1,31)=5.71-8.06]$.

In order to investigate reading span influences on these "switch" effects, the analyses were carried out on the selected group of 12 high- and 12 low-span participants. In this analysis, significant three-way interactions between group, "switch," and dominance were found [Fz, C3, Cz, and C4; $F(1,22)=3.27-12.86]$. Separate analyses were therefore carried out for each span group. The high-span subjects did not show any significant "Switch" $\times$ Dominance interaction indicating that the direction of switch does not matter; they show a clear N400 effect for both "switch" conditions. The analysis for low-span subjects showed significant "Switch" $\times$ Dominance interactions for Fz, C3, Cz, and $\mathrm{C} 4[F(1,11)=10.5-19.72]$. Separate analyses for the DD versus DS conditions showed a clear "switch" effect [Fz, C3, Cz, and C4; $F(1,11)=6.37-17.58]$, whereas the SS versus SD analysis did not show any significant "switch" effect at all.

It is important to know the extent to which each span group has assimilated both types of noun cue. We therefore made a direct comparison of the DD and the SS conditions at the verb and carried out a new analysis having group and DDvsSS as a factor. There was only a main effect of DDvsSS for all tested electrodes $[\mathrm{Fz}, \mathrm{C} 3$, $\mathrm{Cz}, \mathrm{C} 4$, and Pz; $F(1,22)=3.37-13.19$ ] but no interaction

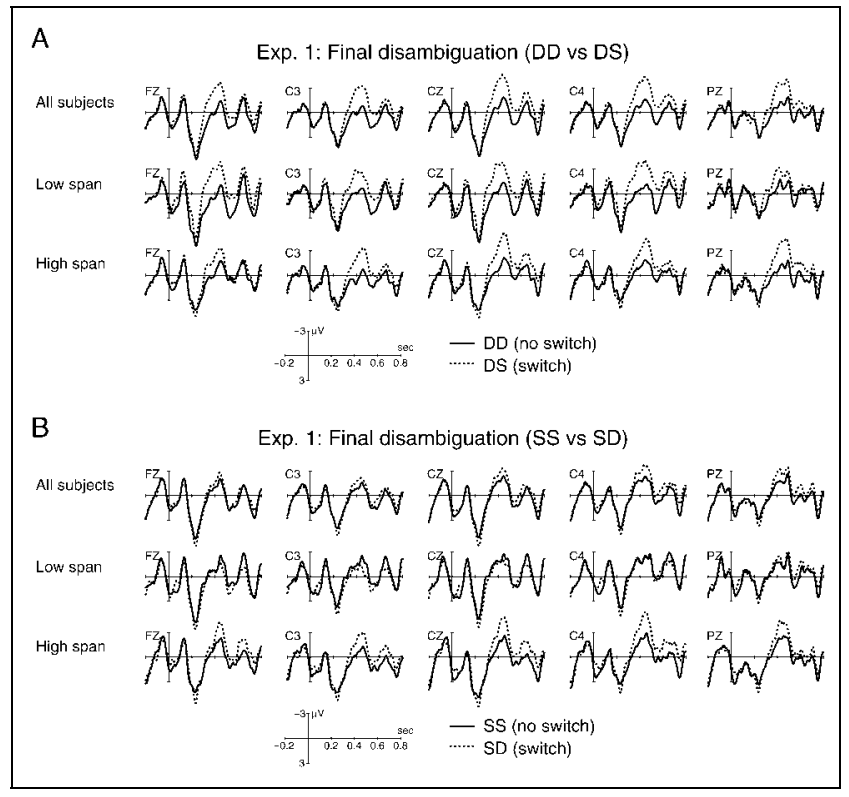

Figure 2. (A) ERPs for the verb position of Experiment 1 where the final disambiguation was given. Disambiguation cue was dominant, the final disambiguation was either dominant (DD, solid line) or subordinate (DS, dashed line). In the DS case a meaning switch had to be performed. (B) ERPs for the verb position of Experiment 1 where the final disambiguation was given. Disambiguation cue was subordinate, the final disambiguation was either subordinate (SS, solid line) or dominant (SD, dashed line). In the SD case a meaning switch had to be performed.

of Group $\times$ DDvsSS $[F(1,22)=0.07-1.30, n s]$. This analysis therefore shows that although the subordinately related verb shows a larger N400 compared to the dominantly related verb, this effect is similar for highand low-span subjects.

In summary, switching to another meaning elicited a larger N400 component compared to the "nonswitch" condition. This indicates that subjects put more effort in semantic integration processes when the other meaning of a homonym was needed. Thus, the participants used the disambiguation cue (noun) such that at the verb position they mainly had one meaning selectively present in WM. Because the switch was accomplished by the subjects, the "irrelevant" meaning is probably not completely deleted from WM. Alternatively, the other meaning is recomputed (reaccessed?) by the system.

The high-span participants used the disambiguation cue very efficiently and flexibly. It did not matter in which direction they had to switch, they showed a similarly large N400 effect. The low-span participants were less flexible. Although the switch from dominant to subordinate was done similarly as by the high spans, switching from subordinate to dominant was difficult for low-span subjects. In this condition, no N400 "switch" effect occurred, indicating that both meanings were similarly present in WM. To put it differently, high-span subjects can suppress the dominant as well as the 
subordinate meaning of a homonym to an equal extent, whereas low-span readers have difficulty suppressing the dominant meaning.

\section{Discussion}

In this experiment, participants were presented with sentences which started off with an ambiguous noun, which was cued towards a particular meaning three words downstream by another noun. The cueing was followed by a verb which in half of the cases disambiguated the meaning of the homonym to the expected (in line with the disambiguation cue) meaning. In the other half of the sentences, the verb disambiguated to the other meaning having the participants switch meanings. At the point of disambiguation, cueing the noun related to the subordinate meaning had a larger N400 compared to the noun related to the dominant meaning. This indicates that the subordinate meaning was more difficult to integrate than the dominant meaning. Thus, the subordinate meaning was less active in WM than the dominant meaning three words (1500 msec) after encountering the homonym. The N400 effects observed at the disambiguation cue seem to depend on reading span. As these effects did not reach significance in the experiment (but see Experiment 2), we refrain from discussing them at this point and postpone the discussion after the presentation of the results of Experiment 2.

Although the information given by the disambiguation cue was at chance level, subjects seemed to have used this information on-line, otherwise no "switch" effects would have been found. Interestingly enough, the "switch" effects depended on the WM capacity of a participant. The switch from dominant to subordinate showed similarly large N400 effects for both high- and low-span participants, indicating that the subordinate disambiguation was highly unexpected (i.e., highly inhibited on the basis of the cue) for all participants. However, while a similar impact of the unexpected verb can be seen in high-span readers when the final (verb based) disambiguation switched from subordinate to dominant, low-span readers, in contrast, did not show any "switch" effect in this condition. Thus, the highspan readers were able to inhibit the irrelevant meaning very quickly and efficiently. The low-span readers, in contrast, seemed to have problems inhibiting the dominant meaning, whereas the inhibition of the subordinate meaning was quite successful. It is important to note that when the sentence followed the cued meaning of the word (i.e., DD and SS), the ERPs did not show any differences at the following verb. If one compares the ERPs for the high- and low-span participants (cf. Figure 2), it is clear that no span difference at the verb was present, which depended on whether the dominant or the subordinate meaning of the homonym was used/cued! This in turn indicated that high- as well as low-span participants had no problem "activating" the cued meaning of the homonym independent of the cueing direction. This makes the data of the "switch" condition extremely informative.

Although low-span subjects clearly show problems with inhibiting irrelevant memory items (i.e., irrelevant meaning), in the present experiment, the question remains as to whether the observed effects are due to an inefficiency in mastering inhibition and activation successfully or whether they are due to the timing of inhibition and activation. If it is due to timing, lowspan readers should do as well as high-span readers if low-span participants are given more time between the disambiguation cue and the final disambiguation. The next experiment was set out to explore this issue by inserting a contextually neutral word, a name of a person, between the disambiguation cue and the final disambiguation (Example: Der Ball wurde vom Spieler Ralf eröfnet; The ball was by the player Ralf opened). Proper names were used because they do not add any extra information regarding the disambiguation but they do add an extra $500 \mathrm{msec}$ of processing time.

\section{EXPERIMENT 2: SWITCHING TIME}

\section{Results}

\section{Percentage Correct}

Overall, participants reached a mean of $90 \%$ correct on the postsentential task. The analysis across all subjects showed a main effect of switch indicating that question answering after the occurrence of a switch condition was less correct [switch: 88\% vs. no-switch: 92\%; $F(1,31)=17.15, p<.0002]$. The analysis carried out on the subgroup of high- and low-span subjects also only showed the main effect of switch $[F(1,22)=$ $11.48, p<.003$ ] but did not reveal a group main effect $[F(1,22)=2.16, n s]$ nor any significant interactions with group. Thus, as in Experiment 1, highand low-span subjects performed similarly on the postsentential task.

\section{ERPs on the Disambiguation Cue}

As can be seen in the upper panel of Figure 3, the data demonstrating the disambiguation cueing of Experiment 1 were replicated in this second study: The noun related to the subordinate meaning showed a larger N400 than the noun related to the dominant meaning of the homonym. Significant main effects of noun were found for Fz, C3, and Cz $[F(1,31)=4.0-5.88]$. Next, an analysis in which WM span was a factor was performed.

As in Experiment 1, no interaction of group with noun was found although the ERPs showed a clear but small difference between both groups in that the high-span participants showed a larger N400 difference for the 


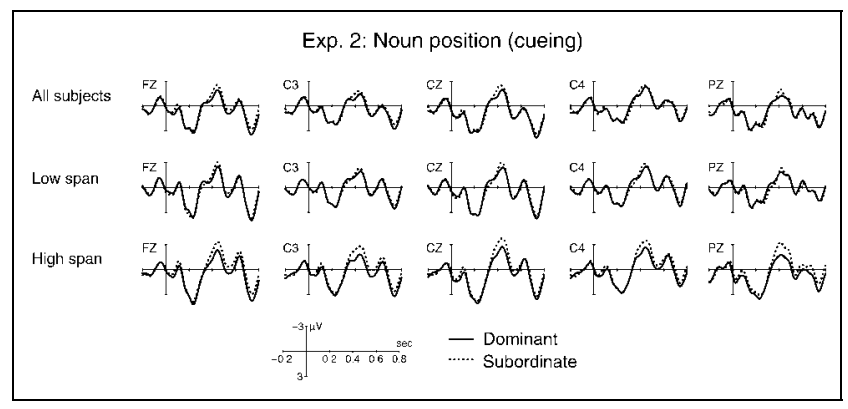

Figure 3. ERPs for the noun position where the disambiguation cue was given. The solid line shows the ERPs elicited by the dominant cueing whereas the dashed line shows the subordinate cueing. The upper row presents the data across all participants of Experiment 2, the middle and lower rows of the pooled data across Experiments 1 and 2 for 24 low- and 24 high-span participants.

subordinate compared to the dominantly related noun. In order to enhance the statistical power to examine the trend seen in Experiments 1 and 2, the data from the two experiments were pooled resulting in 24 subjects in each of the high and low WM span group. As can be seen in the middle and lower panels of Figure 3, which present the pooled ERPs, the high-span compared to the low-span subjects show a much larger N400 for the subordinate noun than for the dominant noun. This observation was confirmed by statistical analyses. Interactions between group and noun were found $[\mathrm{C} 3, \mathrm{Cz}$, Pz; $F(1,46)=4.09-5.73]$. Separate analyses for high- and low-span subjects indicated that the high-span subjects demonstrate robust noun effects on all tested electrodes [Fz, C3, Cz, C4, and Pz; F(1,23) = 8.46-29.48]. The lowspan subjects showed no significant main effect of noun $[F(1,23)=0.15-2.38, n s]$. Thus, as was anticipated on the basis of visual inspection, the analysis of the pooled data showed that high-span subjects maintain the noun related to the subordinate meaning to a lesser extent than the dominantly related noun. Low-span subjects maintain both noun-types to almost the same extent as seen in the marginal N400 difference. It is therefore not unrealistic to suggest that high-span subjects suppress the subordinate meaning more than low-span subjects. Although difficult to explore with the present type of stimuli, it is important to know whether low-span subjects are actually activating any meaning at all. On the one hand, the analyses did not show any main effect of group $[F(1,23)=0.21-1.18]$, indicating that generally speaking, both groups showed similar N400s leading to the conclusion that low spans indeed activated both meanings. In order to establish this suggestion to a larger extent, a post hoc comparison between the dominant noun of high and low span was carried out. There was no significant main effect of group on any of the electrodes $[F(1,23)=0.00-0.15, n s]$, indicating that the N400 for the dominant noun is similar for both groups. Since the N400 for the dominant and subordinate noun was similar within the low-span group, we indirectly confirmed that low span had both meanings active in memory.

\section{ERPs on the Verb}

As should be expected on the basis of Experiment 1, switching between the meanings at the verb gave rise to a larger N400 component than no switching (see Figure 4). As in Experiment 1, switching from dominant to subordinate gave rise to a larger N400 effect compared to switching from subordinate to dominant. Both these observations were confirmed by statistical analyses. Clear main effects of "switch" were found for analyzed electrodes $[F(1,31)=13.6-35.63]$. At the $\mathrm{Pz}$ electrode, an interaction of "switch" with dominance was present $[F(1,31)=6.47]$. This interaction indicates that the N400 "switch" effect for the dominant condition is larger than that for the subordinate condition. Separate analyses were carried out for the dominant (DD vs. DS) and the subordinate (SS vs. SD) conditions. Both the dominant and subordinate conditions showed clear and robust main effects of "switch" [dominant: $F(1,31)=11.26-30.29$; subordinate: $F(1,31)=5.42-$ 15.27]. Thus, although smaller, the N400 "switch" effect was still present in the subordinate condition.

In the main analysis carried out for the high- and low-span subjects, main effects of "switch" were found

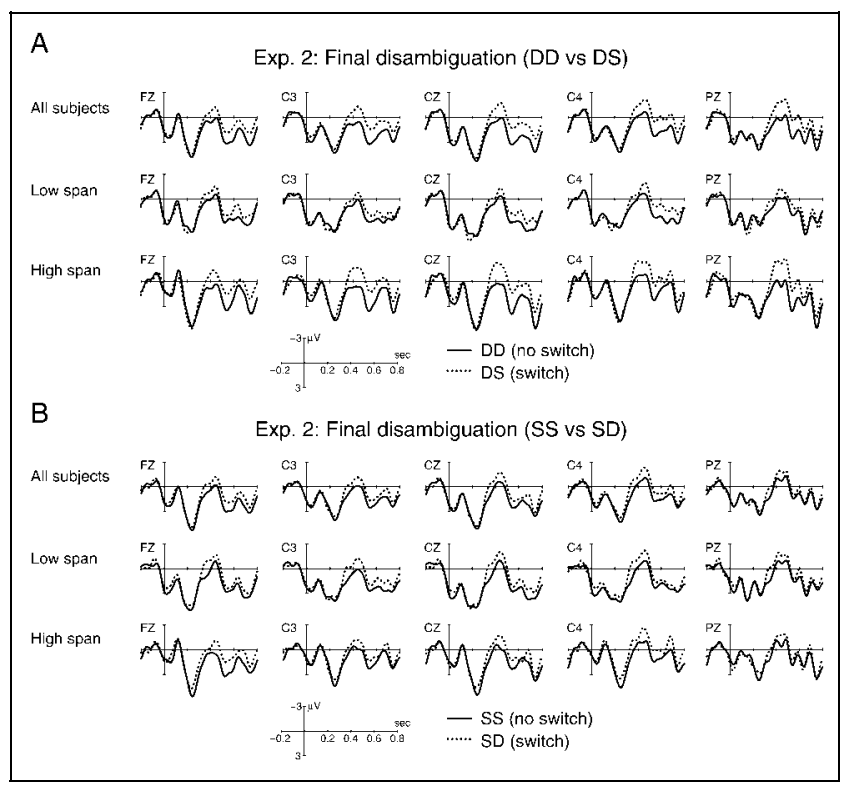

Figure 4. (A) ERPs for the verb position of Experiment 2 where the final disambiguation was given. Cueing was dominant, the final disambiguation was either dominant (DD, solid line) or subordinate (DS, dashed line). In the DS case a meaning switch had to be performed. (B) ERPs for the verb position of Experiment 2 where the final disambiguation was given. Cueing was subordinate, the final disambiguation was either subordinate (SS, solid line) or dominant (SD, dashed line). In the SD case a meaning switch had to be performed. 
for all analyzed electrodes $[\mathrm{Fz}, \mathrm{C} 3, \mathrm{Cz}, \mathrm{C} 4$, and $\mathrm{Pz}$; $F(1,22)=9.14-21.8]$. Three-way interactions between span group, "switch," and dominance were detected at $\mathrm{C} 3$ and $\mathrm{Pz}[F(1,22)=3.95-4.65]$. The step-down analyses for high and low span were therefore carried out for these two electrodes. For the low-span subjects, only main effects of "switch" were found [C3 and Pz; $F(1,11)=3.83-6.44]$. High-span participants showed beside the "switch" main effect [C3 and Pz; $F(1,11)=$ 12.3-18.46] also interactions of "switch" with dominance [C3 and $\mathrm{Pz} ; F(1,11)=3.6-5.37]$, indicating that the "switch" effect was larger in the dominant condition. Thus, low-span participants showed a "switch" effect which was independent of the switch direction. High span showed a larger "switch" effect when they had to switch from dominant to subordinate.

As in Experiment 1, we conducted an analysis having span group and DDvsSS as factors in order to determine how the noun cue was used by each group. At the $\mathrm{Pz}$ electrode, there was a main effect of DDvsSS $[F(1,22)=$ 3.45] and as in Experiment 1, no interactions of group with DDvsSS were found $[F(1,22)=0.1-1.29, n s]$. Thus, as in Experiment 1, these findings also imply that although the subordinately related verb shows a larger N400 compared to the dominantly related verb, this effect is similar for high- and low-span subjects.

\section{Discussion}

The effects found in Experiment 1 on the disambiguation cue were replicated. Generally speaking, the cueing towards the subordinate meaning elicited a larger N400 compared to the dominant condition. This indicates that across all subjects, the noun related to the dominant meaning was easier to integrate and therefore probably more active in WM. The combined analysis across Experiments 1 and 2 for high- and low-span participants demonstrate the influence of WM capacity: The high-span subjects showed a significant larger N400 component for the cueing noun pointing towards the subordinate meaning of the homonym, whereas lowspan subjects did not show a significant difference. This strongly supports the view that high-span participants mainly had the dominant meaning in their WM at the cueing position, whereas low-span participants had both meanings equally active in WM at this point. The data thus appear to support the hypothesis that inhibition is the relevant cognitive process underlying WM (and not activation which would have predicted the reverse effects).

The data related to the meaning switch at the verb were somewhat more complex, however. Recall that in order to give the subjects a bit more time between cueing and final disambiguation, a proper name occupying 500 msec was inserted. This manipulation was made to explore whether additional time would help the low-span subjects to inhibit the dominant meaning to the same extent as the subordinate meaning. The low-span subjects showed a small but significant "switch" effect, which was not affected by the direction of the switch. Thus, the data support the hypothesis that if low-span subjects get more time, they can suppress the dominant meaning as do high-span subjects under less time. Interestingly, when given more time to process, the high-span subjects display a directional bias in their "switch" effect. The effect was much larger when switching from dominant to subordinate than the other way around. Thus, when given more time between the cue and final disambiguation, highspan participants seem to "switch back" to, or "reactivate," the dominant meaning even if the disambiguation cue pointed toward the subordinate interpretation (cf. the SD condition). Such a "reactivation" process can easily be implemented in a processing model assuming ranked activation with a high ranking for the temporarily relevant information and partial suppression of temporarily irrelevant information.

We return now to consider the finding that at the point of cueing the low-span subjects appear to have both meanings present in their WM. Would it be possible for low-span subjects to continue using this memorydemanding strategy (keep both meanings active) if the load on memory was increased by inserting an additional subclause between the ambiguous word and the disambiguation cue? ${ }^{4}$ (Example: Der Ball wurde, als die Sonne schien, vom Spieler eröffnet; The ball was, when the sun shined, by the player opened). This question was addressed in the next experiment.

\section{EXPERIMENT 3: LOADING WM}

\section{Results}

\section{Percentage Correct}

Overall, participants reached a mean of $93 \%$ correct on the postsentential task. The analysis across all subjects showed, as in Experiments 1 and 2, a main effect of switch indicating that question answering after the occurrence of a switch condition was less correct [switch: 91\% vs. no-switch: 95\%; $F(1,28)=35.55, p<$ $.00001]$. An interaction of switch with dominance indicated that the effect of switch was largest when switching from the dominant towards the subordinate (DS) meaning [DD vs. DS: $95 \%$ vs. $90 \%$; SS vs. SD: $95 \%$ vs. $92 \% ; F(1,28)=5.63, p<.025]$.

In contrast to Experiments 1 and 2, the analysis carried out on high- and low-span subjects showed a significant main effect of group indicating that the high-span participants performed 5\% better than the low-span participants [low span: 90\%, high span: $95 \% ; F(1,22)=7.09, p<.014]$. As in the overall analysis, a main effect of switch was found $[F(1,22)=$ 34.9, $p<.0001]$. A significant interaction of group with switch indicated that the switch effect was larger 


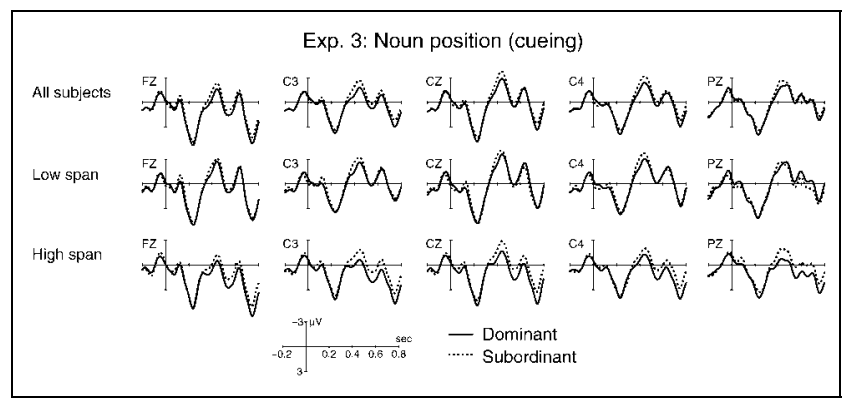

Figure 5. ERPs for the noun position of Experiment 3 where the disambiguation cue was given. The solid line shows the ERPs elicited by the dominant disambiguation cue, whereas the dashed line shows the subordinate disambiguation cue. The upper panel presents the data across all participants, the middle panel of the 12 low-span participants, and the lower panel of the 12 high-span participants.

for low-span subjects [low span: switch: 87\%, no switch: 93\%; high span: switch: 94\%, no-switch: 96\%; $F(1,22)=5.06, p<.035]$. Thus, Experiment 3 revealed WM span-related performance differences in the postsentential task.

\section{ERPs at the Disambiguation Cue}

As can be seen in Figure 5 where the noun position across all subjects is presented, there was a clear and significant N400 difference between the subordinate and the dominant meaning. As in the foregoing experiments, the N400 elicited by the subordinate meaning was larger. The analysis showed significant main effects of noun at Fz, C3, Cz, and C4 $[F(1,28)=4.98-16.35]$.

The overall analysis for both span groups showed significant Group $\times$ Noun interactions in the $\mathrm{N} 400$ region at a posterior electrode [i.e., Pz; $F(1,22)=$ 5.29]. On the basis of this interaction, separate analyses for high- and low-span subjects were carried out. For low-span participants, only C3 showed a main effect of noun $[F(1,11)=4.02]$. The high-span subjects showed a significant noun main effect for all tested electrodes [Fz, C3, Cz, C4, and Pz; F(1,11) = 3.61-9.88]. Thus, low-span subjects appear to have both meanings similarly active in WM, whereas the high-span group appears to have mainly the dominant meaning present. It is interesting to note that compared to high-span subjects, the absolute N400 amplitude is larger in the low-span participants. This amplitude effect was, however, not significant.

\section{ERPs at the Verb}

Across all subjects, there were significant main effects of "switching" context as well as interactions of "switch" with dominance for all tested electrodes [Fz, C3, Cz, C4, Pz; $F(1,28)=7.3-21.27$, respectively, $F(1,28)=3.25-$ 6.51]. Separate analyses for SS versus SD and DD versus DS showed that both had a "switch" effect although it was less pronounced for the SD switch. In this condition, the frontal electrodes did not show any "switch" main effect [DD vs. DS: Fz, C3, Cz, C4, Pz; F(1,28) = 7.86-17.55; SS vs. SD: C3, Cz, Pz; $F(1,28)=4.16-7.96]$.

The main analyses for the high- and low-span subjects showed interactions with the factor group $[\mathrm{Fz}, \mathrm{C} 3, \mathrm{Cz}$, C4, Pz; $F(1,22)=5.43-10.56]$. On the basis of these interactions, separate analyses for high- and low-span subjects were carried out.

In high-span subjects, main effects of "switch" were found [Fz, C3, Cz, C4, Pz; $F(1,11)=4.2-12.57]$ as well as interactions of "switch" with dominance [C4, Pz; $F(1,11)=3.65-4.09$ ]. Low-span subjects only showed a main effect of "switch" $[\mathrm{Pz} ; F(1,11)=4.04]$. The data from the low-span subjects looked like that found in Experiment 1 (i.e., the switch from dominant to subordinate was more difficult than from subordinate to dominant). This impression was, however, not confirmed by the statistical analyses since no interactions between dominance and "switch" were detected. As can be seen in Figure 6, the data of the high-span subjects showed that both "switch" conditions had a similar N400 component. Only the DD condition showed a clearly reduced N400 component, indicating that this condition was most easy to integrate. The N400 for the SS condition almost approached that of both "switch" conditions. This observation was confirmed by the analysis carried out for SSvsDD and group. Clear

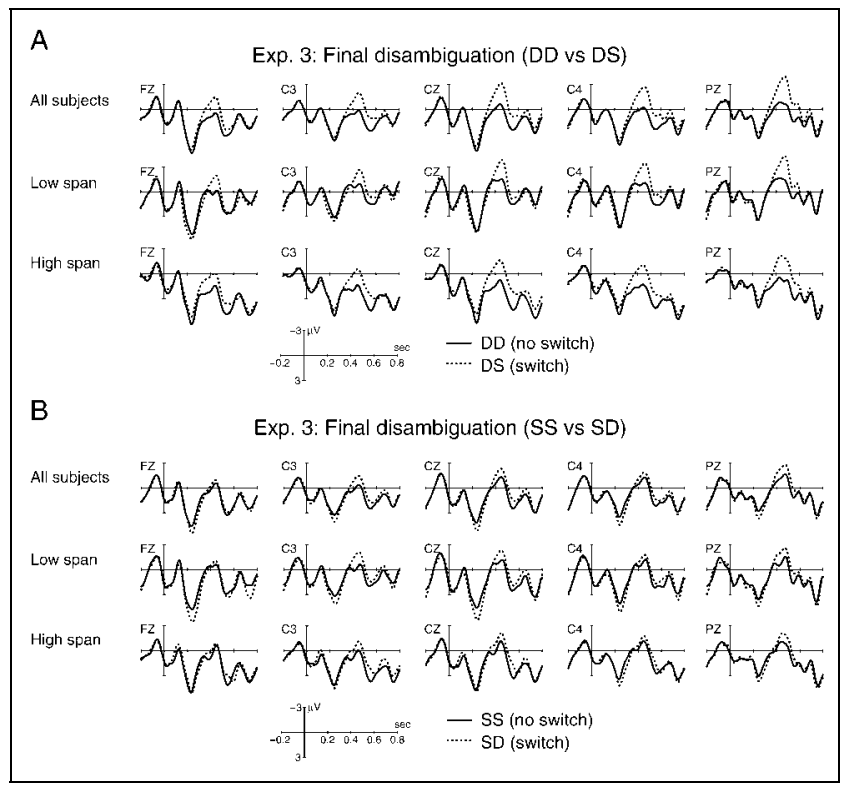

Figure 6. (A) ERPs for the verb position of Experiment 3 where the final disambiguation was given. Cueing was dominant, the final disambiguation was either dominant (DD, solid line) or subordinate (DS, dashed line). In the DS case a meaning switch had to be performed. (B) ERPs for the verb position of Experiment 3 where the final disambiguation was given. Cueing was subordinate, the final disambiguation was either subordinate (SS, solid line) or dominant (SD, dashed line). In the SD case a meaning switch had to be performed. 
interaction of group with DDvsSS was found $[\mathrm{Fz}, \mathrm{C} 3, \mathrm{Cz}$, $\mathrm{C} 4$, and Pz; $F(1,22)=3.16-4.56]$. On the basis of these interactions, separate analyses were performed for highand low-span subjects. The low-span subjects did not show a significant difference between DD and SS. The high span, however, showed clear differences at $\mathrm{Fz}, \mathrm{Cz}$, $\mathrm{C} 4$, and $\mathrm{Pz}[F(1,11)=3.28-6.05]$ in that SS showed a larger N400 as the DD condition.

\section{Discussion}

In Experiment 3, a subclause was added between the homonym and the disambiguation cue in order to test whether or not low-span subjects would be able to hold both meanings of the homonym in WM even when the load on their memory was relatively high. The data showed that indeed the low-span participants were still holding both meanings, whereas the high spans only had the dominant meaning present as in the previous less memory-demanding experiments. In addition, high-span participants showed (in comparison to Experiments 1 and 2) a relatively large N400 effect indicating that they were highly focused on the dominant meaning. Analysis at the verb position also showed this very narrow focus of the high-span participants since the "nonswitch" subordinate condition (SS) gave rise to a very large N400 component, whereas the "nonswitch" dominant condition (DD) did not. The verb position of the low-span subjects also confirmed this strategy: Both the SS and DD conditions showed a similar N400 component.

It is important to note that, in contrast to Experiments 1 and 2, the behavioral data of the postsentential task showed behavioral differences which depended on WM span. High-span participants performed better than lowspan participants and showed a smaller performance decrement in the switch conditions. These behavioral data, in combination with null effects of WM span in Experiments 1 and 2, indicate that inserting a subclause (which made the sentence structure much more complex) did load WM to a larger extent than the insertion of a proper name in Experiment 2.

\section{GENERAL DISCUSSION}

The main goal of this study was to specify those WM mechanisms that underlie efficient language processing at the sentence level. Sentences containing ambiguous words were used to decide between different proposals that have been formulated to account for processing efficiency. Two strongly opposing views were under investigation: equally strong activation of both meanings of an ambiguous word versus inhibition of the irrelevant meaning (i.e., activation vs. inhibition hypothesis). All three experiments showed clear evidence against the view that activational processes are playing a major role during the efficient processing of ambiguous sentence material for high-span readers. The data rather suggest that inhibition, or at least a reduced activation of the irrelevant meaning, is the underlying mechanism. In Experiments 1 and 2, the noun which was used for cueing showed a significantly larger N400 for the subordinate-related than for the dominant-related noun for high-span subjects. This N400 effect was not found for low-span participants. Thus, the presence of an N400 effect for the high-span participants indicated that the noun related to the subordinate meaning was more difficult to integrate than the noun related to the dominant meaning. This pattern of result can be interpreted as an indication that the dominant meaning is largely present in WM for high-span subjects and that the subordinate meaning is suppressed to a great extent. Low-span participants can integrate both the dominantand subordinate-related noun equally well and therefore appear to have both meanings equally present in their WM. These results are in line with inhibition as the mechanism underlying WM, supporting the processing of ambiguous words (cf. Gersbacher \& Faust, 1991), and not with the activation hypothesis (cf. Miyake et al., 1994). The data of Experiment 3 strengthen this view. In this experiment, we lengthened the distance between the homonym and the disambiguation cue by inserting a neutral subclause. Low-span subjects still showed no significant N400 difference for the dominant and subordinate noun, indicating that they still have both meanings actively present in their WM. The high-span participants, however, displayed a clear N400 effect in that the N400 amplitude was larger for the subordinate disambiguation cue than for the dominant one, indicating suppression of the subordinate meaning. This N400 effect was larger than those found in Experiments 1 and 2, suggesting that the lengthening of the neutral region resulted in an enhanced focusing of the WM content for the high-span participants. Note that we cannot exclude the possibility that for high-span subjects the subordinate meaning simply decays in time and is therefore less active in memory. What is clear, however, is that highspan subjects, in contrast to low-span subjects, do not actively keep the subordinate meaning active in WM.

The next issue is related to the flexibility of the WM resource. After subjects were presented with the disambiguation cue, a final disambiguation was provided by a verb. In half of the cases, the cueing and the final disambiguation did not match, requiring subjects to switch meaning. Those "switch" trials gave rise to a larger N400 compared to the "nonswitch" trials. This in itself is interesting since it demonstrates that the disambiguation cue was used by the subjects to solve the ambiguity. Experiment 1 showed that both high- as well as low-span subjects can switch between the two meanings. However, whereas there was no directional effect for high-span subjects, low-span subjects showed no N400 effect when switching from subordinate to dominant. One can therefore conclude that low-span subjects 
had problems inhibiting the dominant meaning. These data also indicate that high-span subjects are able to use their WM in a more flexible and rapid manner than lowspan subjects. As shown in Experiment 2, low-span subjects are able to suppress the dominant meaning when they have additional time to do so.

Taking all three experiments into account, the situation involved in processing of homonyms is as follows: High-span subjects read a homonym and activate (probably) both meanings to a large extent. By the time they arrive at the disambiguation cue, they have suppressed the subordinate meaning. The longer the unbiased passage, the stronger is the suppression. If the disambiguation cue indicates that a subordinate meaning is probably correct, they shift the activation levels of the two meanings very swiftly to the subordinate meaning but only for a short duration. If the sentence continues and does not give any additional confirmation that their switch towards the subordinate meaning was correct, they switch back to the dominant meaning and then run into trouble when the sentence turns out to require a subordinate meaning. Low-span subjects read the homonym and activate both meanings. When they arrive at the disambiguation cue they have both meanings present in WM. This is even the case when WM is loaded by an extension to the neutral part of the sentence (Experiment 3). If the disambiguation cue indicates that they probably have to switch meanings, they can do so when sufficient time is given; however, if a fast response is needed, they have problems suppressing the dominant meaning.

In this series of experiments, we have shown that inhibitory/suppression processes play a crucial role in WM supporting language comprehension. High-span subjects, shown to be more efficient in language processing in a number of studies, can use their inhibition/ suppression in a very flexible manner although they tend to stick to one meaning. Low-span subjects, shown to be less efficient in language processing in a number of studies, stick to both meanings. Keeping several meanings active may be the underlying reason on why low spans are slower than high-span subjects in sentence processing. It is the temporary suppression of irrelevant information that may guarantee efficient language comprehension.

\section{METHODS}

\section{Sentence Material}

Before the actual experimental sentences were chosen, a large rating study was carried out. This began with the choice of approximately 300 homonyms by the experimenters. A cloze procedure, in which 100 students were asked to complete sentences such as Der Ball wurde vom ... (The ball was by the ...) with a noun and a verb (e.g., Kind geworfen; child thrown), was run using these homonyms. After having completed the cloze continuation on all words, the participants were told that the words in the sentences were (as they might have seen themselves) ambiguous and that they should fill out the form a second time using the "other" meaning. On the basis of this procedure, items were chosen which had exactly two meanings of which at least $95 \%$ of the participants mentioned one meaning first, hereafter termed the dominant one. This cloze procedure was also used to rate the expectancy of the noun. Only items with a cloze probability of $80 \%$ or more for a specific noun (in either meaning of the ambiguous word) was used in the next rating. In this second cloze procedure, 50 new participants were used to verify whether the verbs were highly expected. The participants were required to end sentences such as Der Ton wurde vom Sänger/Töpfer ... (The tone/clay was by the singer/potter...) with a verb. Items having a cloze probability of $80 \%$ or more were kept in the materials. Finally, a plausibility check was performed in which another 50 participants had to explicitly rate the complete sentence with respect to the use of the ambiguous word in its dominant or subordinate meaning (Der Ton wurde vom Sänger gesungen). At the end of this extensive procedure, we were left with 91 items of which 88 were used for the experiment. In order to prevent sentence final effects, an additional subclause of four words was added to the experimental part of the sentence. In order to balance out the expectancy for a meaning switch on the verb of the experimental sentences, filler items with an ambiguous second word were constructed which had the same structure as the experimental items used in a specific experiment but never switched in meaning on the verb.

All sentences were visually presented in an RSVP format with 300 msec per word and a blank screen of 200 msec between the words. The first word of a sentence was preceded by a fixation cross of $300 \mathrm{msec}$. After the presentation of the sentence ending (period included), a blank screen of $700 \mathrm{msec}$ and a "yes-no" question regarding the content of the sentence (5000 msec maximal) were presented. Subjects were instructed that accuracy was more important than speed of the response. A feedback stimulus was presented for 700 msec immediately after the occurrence of a response. Then, after the presentation of a blank screen (300 msec), the next sentence started. Word length was always kept within $2^{\circ}$ of the visual field. The words, which had a height of approximately $0.4^{\circ}$, were presented in black on a gray background.

\section{Presentation Sequence}

All experiments were conducted in two sessions with a minimum time interval of 1 week between sessions. In each session subjects received a small training block followed by two blocks which had 88 experimental sentences and 88 fillers each. The four types of stimuli 
were equally distributed in a block (22 of each). Between the blocks a break of 15 min was given in which subjects were allowed to read a magazine of their choice. None of the sentences used in the training were experimental sentences. Each experimental sentence was presented in each of the four conditions (i.e., A-D; see below). Since only two sessions were feasible, a repetition of an experimental sentence within one session was inevitable. In order to have the least problems of item repetition (cf. Besson \& Kutas, 1993), we presented the experimental items in such a way that either versions A and D or versions $\mathrm{B}$ and $\mathrm{C}$ of a particular sentence were present in a particular block of a particular participant. When a DD condition was presented in the first block, it was replaced by an SD condition in the second block; SS replaced by DS, SD replaced by DD, and DS replaced by SS (see below). The initial type of a particular sentence was pseudorandomized making sure that all conditions were equally frequent within a block. Of each block, four versions were made which permutated all four sentence types. Each of the versions was administered to a total of 8 persons. Thus, each participant received two sessions in which a total of 352 experimental and 352 filler sentences were presented.

\section{Procedure}

A session lasted approximately $3 \mathrm{hr}$. Participants were seated in a dimly lit room, facing a color video screen at a distance of $100 \mathrm{~cm}$. When necessary, instructions were given to blink only when the fixation cross appeared.

\section{Recordings}

The electroencephalogram (EEG) was recorded with 59 $\mathrm{Ag}-\mathrm{AgCl}$ electrodes (electrocap) from $\mathrm{Fp} 1, \mathrm{FpZ}, \mathrm{Fp} 2$, Af7, Af3, AfZ, Af4, Af8, F7, F5, F3, Fz, F4, F6, F8, Ft7, Fc5, Fc3, Fcz, Fc4, Fc6, Ft8, Ft7, Fc5, Fc3, Fcz, Fc4, Fc6, Ft8, T7, C5, C3, Cz, C4, C6, T8, Tp7, Cp5, Cp3, CpZ, Cp4, Cp6, Tp8, P7, P5, P3, Pz, P4, P6, P8, Po7, Po3, Poz, Po4, $\mathrm{Po} 8, \mathrm{O} 1, \mathrm{Oz}, \mathrm{O} 1$, and the left mastoid each referred to the right mastoid (nomenclature as proposed by the American Electroencephalographic Society, 1991). Bipolar horizontal EOG was recorded between electrodes at the outer left and right canthus. Bipolar vertical EOG was recorded between electrodes above and below the subject's right eye. Electrode resistance was kept under $5 \mathrm{~K} \Omega$. The signals were recorded continuously with a bandpass between DC and $70 \mathrm{~Hz}$ and digitized at a rate of $250 \mathrm{~Hz}$.

\section{Data Analysis}

Average ERPs, starting $200 \mathrm{msec}$ before and lasting $1000 \mathrm{msec}$ after the presentation of the disambiguation cue and its following verb were computed for each electrode position for each of the conditions.
Trials containing ocular and amplifier saturation artifacts (EOG rejection $\pm 50 \mu \mathrm{V}$ ) were excluded from the averages. Averages were aligned to a 200-msec prestimulus baseline. In order to describe the onsets and length of the ERP effects in reasonable detail, an analysis was carried out in which the data were statistically evaluated using a latency window between 350 and $450 \mathrm{msec}$. This latency window, which is clearly incorporating the $\mathrm{N} 400$ maximum, reflects N400 activity reliably.

All dependent variables were quantified using repeated-measure ANOVAs. In the analysis done on the noun, the within-subjects variables were session (2) and noun-type (dominant vs. subordinate). In the analyses on the verbs, an additional within-subjects variable called "switch" (2) was included. The analyses on both noun and verb were also done with WM span (2) as a between-subjects factor using the subset of high and low WM span subjects. The analyses of the interval data were carried out for a reduced set of electrodes (Fz, C3, Cz, $\mathrm{C} 4$, and $\mathrm{Pz}$ ). The Geisser-Greenhouse correction (Vasey \& Thayer, 1987; Greenhouse \& Geisser, 1959) was always applied when evaluating effects with more than one degree of freedom in the numerator.

\section{Experiment 1}

\section{Participants and Data Analysis}

Thirty-two native German-speaking students (16 women, mean age 24.8 years, age range $19-30$ years) were paid approximately $50 \mathrm{DM}$ for their participation. All participants were right-handed and had normal or correctedto-normal vision. WM span was measured for each participant on the basis of a German version of the Daneman and Carpenter (1980) reading span test (see also Vos et al., 2001). Out of the 32 participants, two groups of 12 subjects were selected which had either a high (4.0-6.0; mean 4.4) or a low (2.0-3.5; mean 2.8) WM span. Approximately $6 \%$ of the trials were excluded from the averages due to ocular and amplifier saturation artifacts.

\section{Stimuli}

A total of 88 experimental sentences were constructed containing 10 words each. The German sentences had an ambiguous word on the second position. The ambiguous noun was followed by a nominal disambiguation cue at the fifth word and a final disambiguating verb at sixth word position. The experiment included 88 filler sentences, each containing one ambiguous word. The experimental sentences were manipulated in such a way that the lexical elements used for the disambiguation cue (nouns) as well as the final disambiguation (verbs) had a high cloze probability (mean: 93 \%; see Taylor, 1953). A 2 (noun dominant/ subordinate $) \times 2$ (verb dominant/subordinate) design 
was used. Examples with literal English translation are presented below:

Ambiguous word: Ton (either tone or clay)

A Der Ton wurde vom Sänger gesungen, als ... (The tone was by the singer sung, when ...)

Dominant disambiguation cue, Dominant disambiguation (DD)

B Der Ton wurde vom Töpfer gebrannt, weil ...

(The clay was by the potter baked, because...)

Subordinate disambiguation cue, Subordinate disambiguation (SS)

C Der Ton wurde vom Sänger gebrannt, obwohl ... (The clay was by the singer baked, although...)

Dominant disambiguation cue, Subordinate disambiguation (DS)

D Der Ton wurde vom Töpfer gesungen, während ... (The tone was by the potter sung, while ...)

Subordinate disambiguation cue, Dominant disambiguation (SD)

\section{Experiment 2}

\section{Participants and Data Analysis}

Thirty-two native German-speaking students (18 women, mean 23.5 years, age range 19-29 years) were paid approximately $50 \mathrm{DM}$ for their participation. All participants were right-handed and had normal or corrected-to-normal vision and did not participate in Experiment 1. WM span was measured for each participant on the basis of a German version of the Daneman and Carpenter (1980) reading span test. Out of the 32 participants, two groups of 12 subjects were selected which had either a high (5.4) of a low (2.9) WM span. Approximately $5.5 \%$ of the trials were excluded from the averages due to ocular and amplifier saturation artifacts.

\section{Stimuli}

The 88 experimental sentences as discussed in Experiment 1 were changed in that a proper name was inserted between the disambiguation cue and the final disambiguation. Nothing else was changed.

Ambiguous word: Ton (either tone or clay)

A Der Ton wurde vom Sänger Harry gesungen, als ... (The tone was by the singer Harry sung, when ...)

Dominant disambiguation cue, Dominant disambiguation (DD)

B Der Ton wurde vom Töpfer Peter gebrannt, weil ... (The clay was by the potter Peter baked, because...)

Subordinate disambiguation cue, Subordinate disambiguation (SS)

C Der Ton wurde vom Sänger Harry gebrannt, obwohl ... (The clay was by the singer Harry baked, although ...)

Dominant disambiguation cue, Subordinate disambiguation (DS)
D Der Ton wurde vom Töpfer Peter gesungen, während ... (The tone was by the potter Peter sung, while ...)

Subordinate disambiguation cue,

Dominant disambiguation (SD)

All sentences had the same presentation format as in Experiment 1. Filler items were adapted in a similar way as the experimental items.

\section{Experiment 3}

\section{Participants and Data Analysis}

Twenty-nine native German-speaking students (17 women, mean 23.8 years, age range 19-29 years) were paid approximately $50 \mathrm{DM}$ for their participation. All participants were right-handed and had normal or corrected-to-normal vision and did not participate in Experiment 1 or 2. WM span was measured for each participant on the basis of a German version of the Daneman and Carpenter (1980) reading span test. Out of the 29 participants, two groups of 12 subjects were selected which had either a high (5.5) or a low (2.8) WM span. Approximately $9.4 \%$ of the trials were excluded from the averages due to ocular and amplifier saturation artifacts.

\section{Stimuli}

The 88 experimental sentences as discussed in Experiment 1 were changed in that a subclause, which was neutral with regard to the meaning of the homonym, was added between the homonym and the disambiguation cue. Nothing else was changed.

Ambiguous word: Ton (either tone or clay)

A Der Ton wurde, als die Sonne schien,vom Sänger gesungen, als ...

(The tone was, when the sun shined, by the singer sung, when ...)

Dominant disambiguation cue, Dominant disambiguation (DD)

B Der Ton wurde, als die Sonne schien,vom Töpfer gebrannt, weil ...

(The clay was, when the sun shined, by the potter baked, because...)

Subordinate disambiguation cue, Subordinate disambiguation (SS)

C Der Ton wurde, als die Sonne schien, vom Sängergebrannt, obwobl ... (The clay was, when the sun shined, by the singer baked, although ...)

Dominant disambiguation cue, Subordinate disambiguation (DS)

D Der Ton wurde, als die Sonne schien,vom Töpfer gesungen, wäbrend ... (The tone was, when the sun shined, by the potter sung, while ...)

Subordinate disambiguation cue, Dominant disambiguation (SD) 
All sentences had the same presentation format as in Experiment 1. Filler items were adapted in a similar way as the experimental items.

\section{Acknowledgments}

This work was supported by the Deutsche Forschungsgemeinschaft (FOR 309/1-2). We thank Frank Meyer for his technical support, Silke Urban and Peter Naumann for preparing the stimuli, and Sabine Koitzsch for the data acquisition. We are indebted to Axel Mecklinger and David Swinney for helpful comments on earlier drafts of this manuscript.

Reprint requests should be sent to Thomas C. Gunter, Max Planck Institute of Cognitive Neuroscience, Stephanstrasse 1a, 04103 Leipzig, Germany, or via e-mail: Gunter@cns.mpg.de.

\section{Notes}

1. Note that several theories exist on how prior disambiguating information has its influence on the initial lexical access of an ambiguous word. The "autonomous access" model assumes no influence of prior context in that both meanings will be accessed. A variant of it is the "integration model," which assumes that both meanings are accessed with a priority towards the dominant one. The "reordered access" model assumes an influence such that the appropriate meaning of the word will be more available. The "selective access" model assumes that only the contextually appropriate meaning is available. In the present study, we will not give any prior disambiguating information. We assume based on the vast empirical evidence in the literature that both meanings will, at least directly after word access, be present in this condition. 2. Note that the initial activation level of a particular meaning and its decay function over time also plays an important role in a neutral context.

3. Note that N400 effects also depend on the expectancy of a word at a certain position in a sentence. The higher the expectancy, as measured by, for instance, a cloze procedure, the smaller the N400 will be (cf. Van Petten, 1995). We therefore made sure that the expectancy of the nouns related to either meaning was similar.

4. Note that if we would find a larger $\mathrm{N} 400$ for the subordinated predisambiguation, this also could mean that the subordinate meaning was decayed due to time passing by If not, however, it would confirm the hypothesis that low span "actively" keep both meanings in WM.

\section{REFERENCES}

American Electroencephalographic Society (1991). Guidelines for standard electrode position nomenclature. Journal of Clinical Neurophysiology, 8, 200-202.

Besson, M., \& Kutas, M. (1993). The many facets of repetition A cued-recall and event-related potential analysis of repeating words in same versus different sentence contexts. Journal of Experimental Psychology: Learning, Memory, and Cognition, 5, 1115-1133.

Brown, C. M., \& Hagoort, P. (1993). The processing nature of the N400: Evidence from masked priming. Journal of Cognitive Neuroscience, 5, 34-44.

Caplan, D., \& Waters, G. S. (1999). Verbal working memory and sentence comprehension. Behavioral and Brain Science, 22, $114-126$

Cooke, A., Zurif, E. B., DeVita C., Alsop, D., Koenig, P., Detre, J., Gee, J., Pinango, M., Balogh, J., \& Grossman, M. (2001).
Neural basis for sentence comprehension: Grammatical and short-term memory components. Human Brain Mapping, $15,80-94$

Daneman, M., \& Carpenter, P. A. (1980). Individual differences in working memory and reading. Journal of Verbal Learning and Verbal Behavior, 19, 561-584.

Dopkins, S., Morris, R. K., \& Rayner, K. (1992). Lexical ambiguity and eye fixations in reading: A test of competing models of lexical ambiguity resolution. Journal of Memory and Language, 31, 461-476.

Engle, R. W. (1996). Working memory and retrieval: An inhibition-resource approach. In J. T. E. Richardson, R. W. Engle, L. Hasher, R. H. Logie, E. R. Stoltzfus, \& R. T. Sacks (Eds.), Working memory and buman cognition (pp. 89-119). New York: Oxford University Press.

Fiebach, C. J., Schlesewsky, M., \& Friederici, A. D. (2001). Syntactic working memory and the establishment of filler-gap dependencies: Insights from ERPs and fMRI. Journal of Psycholinguistic Research, 30, 321-337.

Friederici, A. D., Steinhauer, K., Mecklinger, A., \& Meyer, M. (1998). Working memory constraints on syntactic ambiguity resolution as revealed by electrical brain responses. Biological Psychology, 47, 193-221.

Gernsbacher, M. A., \& Faust, M. (1991). The role of suppression in sentence comprehension. In G. B. Simpson (Ed.), Understanding word and sentence (pp. 97-128). New York: Elsevier.

Greenhouse, S., \& Geisser, S. (1959). On methods in the analysis of profile data. Psychometrika, 24, 95-112.

Gunter, T. C., Jackson, J. L., \& Mulder, G. (1995). Language, memory, and aging: An electrophysiological exploration of the N400 during reading of memory-demanding sentences. Psychophysiology, 32, 215-229.

Hagoort, P., \& Brown, C. (1994). Brain responses to lexical ambiguity resolution and parsing. In C. Clifton, L. Frazier, \& K. Rayner (Eds.), Perspectives on sentence processing (pp. 45-80). Hillsdale, NJ: Erlbaum.

Hickok, G. (1993). Parallel parsing: Evidence from reactivation in garden-path sentences. Journal of Psycholinguistic Research, 22, 239-250.

Just, M. A., \& Carpenter, P. A. (1992). A capacity theory of comprehension: Individual differences in working memory. Psychological Review, 99, 122-149.

Just, M. A., Carpenter, P. A., Keller, T. A., Eddy, W. F., \& Thulborn, K. R. (1996). Brain activation modulated by sentence comprehension. Science, 274, 114-116.

King, J., \& Just, M. A. (1991). Individual differences in syntactic processing: The role of working memory. Journal of Memory and Language, 30, 580-602.

King, J., \& Kutas, M. (1995). Who did what and when? Using word- and clause-related ERPs to monitor working memory usage in reading. Journal of Cognitive Neuroscience, 7, $378-397$.

Kutas, M., \& Federmeier, K. D. (2000). Electrophysiology reveals semantic memory use in language comprehension. Trends in Cognitive Sciences, 4, 463-470.

Kutas, M., \& Hillyard, S. A. (1980). Event-related brain potentials to semantically inappropriate and surprisingly large words. Biological Psychology, 11, 99-116.

Mecklinger, A., Schriefers, H., Steinhauer, K., \& Friederici, A. D. (1995). Processing relative clauses varying on syntactic and semantic dimensions: An analysis with event-related potentials. Memory and Cognition, 23, 477-494.

Miyake, A., Just, M. A., \& Carpenter, A. (1994). Working memory constraints on the resolution of lexical ambiguity: Maintaining multiple interpretations in neutral contexts. Journal of Memory and Language, 33, 175-202. 
Rayner, K., \& Frazier, L. (1989). Selection mechanisms in reading lexically ambiguous words. Journal of Experimental Psychology: Learning, Memory, and Cognition, 15 779-790.

Simpson, G. B. (1994). Context and the processing of ambiguous words. In M. A. Gernsbacher (Ed.), Handbook of psycholinguistics (pp. 359-374). New York: Academic Press.

Stowe, L. A., Broere, C. A. J., Paans, A. M. J., Wijers, A. A., Mulder, G., Vaalburg, W., \& Zwarts, F. (1998). Localizing components of a complex task: Sentence processing and working memory. NeuroReport, 9, 2995-2999.

Swaab, T., Brown, C., \& Hagoort, P. (1997). Electrophysiological studies of comprehension deficits in aphasia. International Journal of Psychophysiology, 25, 71-72.

Swinney, D. A. (1979). Lexical access during sentence comprehension: (Re)Consideration of context effects. Journal of Verbal Learning and Verbal Behavior, 18, 645-659.

Taylor, W. L. (1953). "Cloze" procedure: A new tool for measuring readability. Journalism Quarterly, 30, 415-417.
Van Petten, C. (1995). Words and sentences: Event-related brain potential measures. Pschophysiology, 32, 511-525.

Van Petten, C., \& Kutas, M. (1987). Ambiguous words in context: An event-related potential analysis of the time course of meaning activation. Journal of Memory and Language, 26, 188-208.

Vasey, M. W., \& Thayer, J. F. (1987). The continuing problem of false positives in repeated measures ANOVA in psychophysiology: A multivariate solution. Psychophysiology, 4, 479-486.

Vos, S. H., \& Friederici, A. D. (2003). Intersentential syntactic context effects on comprehension: The role of working memory. Cognitive Brain Research, 16, 111-122.

Vos, S. H., Gunter, T. C., Kolk, H. H. J., \& Mulder, G. (2001). Working memory constraints on syntactic processing: An electrophysiological investigation. Psychophysiology, 38, $41-63$.

Vos, S. H., Gunter, T. C., Schriefers, H., \& Friederici, A. D. (2001). Syntactic parsing and working memory: The effects of syntactic complexity, reading span, and concurrent load. Language and Cognitive Processes, 16, 65-103. 